\title{
Echocardiographic features of indirect Gerbode defect in a cat
}

\author{
Sang-Kwon Lee ${ }^{1}$, Namsoon Lee ${ }^{2,3}$, Kyoung-Oh Cho ${ }^{1}$, Mahmoud Soliman ${ }^{1}$, Munsu Yun ${ }^{3}$, Jihye Choi ${ }^{1, *}$ \\ ${ }^{1}$ College of Veterinary Medicine and BK 21 Plus Project Team, Chonnam National University, Gwangju 61186, Korea \\ ${ }^{2}$ College of Veterinary Medicine, Seoul National University, Seoul 08826, Korea \\ ${ }^{3}$ Time Animal Medical Center, Daejeon 35233, Korea
}

\begin{abstract}
This report describes the echocardiographic features of an indirect Gerbode defect in a young cat. Echocardiography revealed high-velocity, turbulent systolic flow directed from left ventricle to right ventricle through a ventricular septal defect. The flow immediately entered the right atrium through a tricuspid septal leaflet. The indirect-type Gerbode defect was confirmed through necropsy. When a high-velocity turbulent flow in the RA without pulmonary hypertension is observed on echocardiography, Gerbode defect should be considered.
\end{abstract}

Keywords: complex cardiac malformation, feline, perimembranous ventricular septal defect, tricuspid leaflet defect

\section{*Corresponding author}

Jihye Choi

College of Veterinary Medicine, Chonnam National University, 77 Yongbong-ro, Bukgu, Gwangju 61186, Korea

Tel: $+82-62-530-2821$

Fax: +82-62-530-2809

E-mail: imsono@chonnam.ac.kr

Sang-Kwon Lee and Namsoon Lee contributed to this work equally as first authors.

ORCID:

Sang-Kwon Lee:

https://orcid.org/0000-0002-3097-0345

Namsoon Lee:

https://orcid.org/0000-0002-9091-6957

Kyoung-Oh Cho:

https://orcid.org/0000-0002-5477-7021

Mahmoud Soliman:

https://orcid.org/0000-0003-4110-3703

Munsu Yun:

https://orcid.org/0000-0002-0483-8084

Jihye Choi:

https://orcid.org/0000-0002-1258-7158

Conflict of Interest

The authors declare no conflicts of interest.

Received: March 5, 2019

Revised: May 9, 2019

Accepted: May 23, 2019
Gerbode defect is a rare type of ventricular septal defect that results in communication between the left ventricle (LV) and the right atrium (RA). Because the tricuspid valve (TV) attaches to the membranous septum more apically than that of the mitral valve, a congenital defect involving the atrioventricular portion of interventricular septum can cause an abnormal communication between the LV and RA [1,2]. The TV malformation, such as a perforation, cleft, widened commissural space, or abnormal chordae, when accompanied by a ventricular septal defect (VSD), also permits the LV to communicate with the RA [1]. This report describes the first case of an indirect Gerbode defect in a young cat.

A 5-month-old, intact female, Korean short-haired cat weighing $1.4 \mathrm{~kg}$ presented with depression, anorexia, and dyspnea for 3 days. Cardiac auscultation was difficult because of tachypnea. One month prior, the cat was involved in a traffic accident, but its vital signs were within the normal range including a normal heart sound.

Thoracic radiography revealed mild cardiomegaly (vertebral heart score: 8.4; reference range: 6.7-8.1) with bilateral atrial dilation and widening of the cranial mediastinum (Fig. 1). Although there was no distinct pulmonary infiltration on radiography, tachypnea was slightly improved and cardiomegaly and cranial mediastinal widening disappeared after intravenous infusion of furosemide (Lasix, Handok Pharm, Korea) at $0.4 \mathrm{~mL} / \mathrm{kg} / \mathrm{h}$ for $36 \mathrm{~h}$. Systolic blood pressure was $95 \mathrm{mmHg}$.

On echocardiography, the RA and right ventricle (RV) were markedly dilated (Fig. 2). A turbulent jet from the base of the tricuspid septal leaflet and not between the TVs flowed into the RA (Fig. 3). Close observation revealed that the turbulent jet initially flowed from the LV to the RV through the upper membranous septum and then it immediately flowed into the RA through the tricuspid septal leaflet. The velocity of the turbulent flow was $3.77 \mathrm{~m} / \mathrm{sec}$ and the pressure gradient was estimated to be $57 \mathrm{mmHg}$. There was no evidence of pulmonary hypertension including RV hypertrophy, pulmonary insufficiency, or dilated pulmonary artery. The flow velocities and Doppler profile of the pulmonary arterial flow were normal. Interventricular septum flattening or paradoxical movement was not detected. LV to RA communication accompanied by a TV defect was tentatively diagnosed. The cat was monitored without medication at the owner's request because clinical signs had disappeared. 


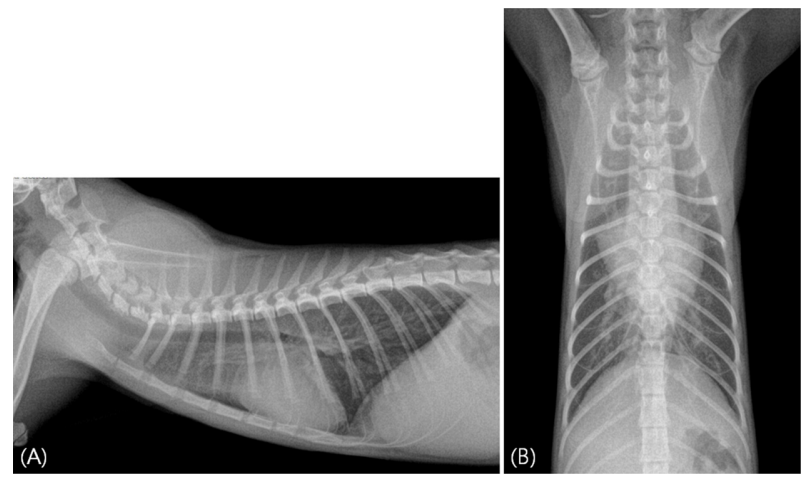

Fig. 1. Right lateral (A) and ventrodorsal (B) radiographs revealed cardiomegaly with bilateral atrial dilation and uniform cranial mediastinal widening.

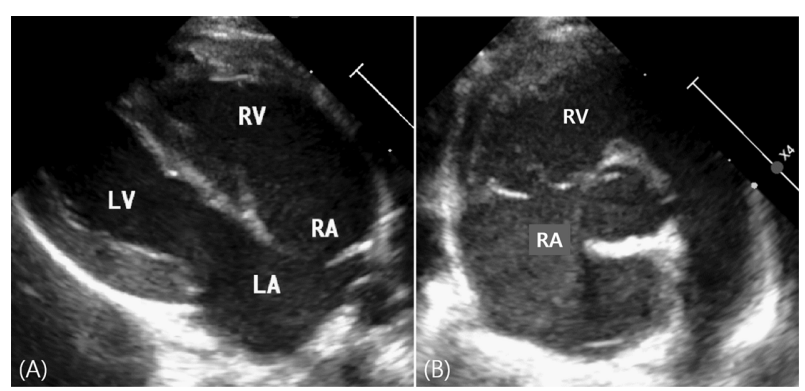

Fig. 2. Right long axis view (A) and right short axis (B) echocardiographic views. RA and RV are markedly dilated. TV shows normal morphology and position in systole (B).

LA, left atrium; LV, left ventricle; RA, right atrium; RV, right ventricle.

Two months later, the cat died after exhibiting neurologic signs such as depression, hyperexcitability, and tremor. On necropsy, a membranous interventricular septum originating in the LV outflow tract just proximal to the aortic valve and a hole in the tricuspid septal leaflet were observed (Fig. 4). The diagnosis was confirmed as indirect-type (type 2) Gerbode defect.
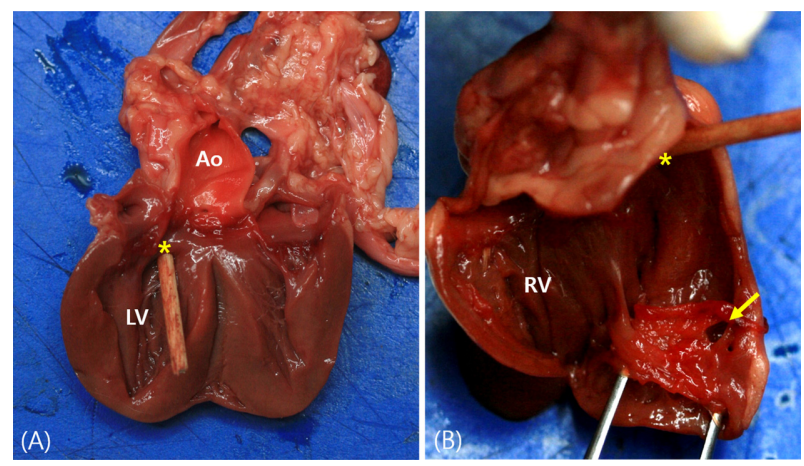

Fig. 4. Necropsy findings; LV (A) and RV (B) in the study cat. There was a tract communicating between the LV and the RV, through the membranous ventricular septum (asterisk). There was also a perforation in the tricuspid septal leaflet (arrow). Ao, aorta; LV, left ventricle; RV, right ventricle.

In indirect-type Gerbode defect, blood flows from the LV through the VSD into the RV and then through a defect in the TV into the RA [1]. A Gerbode defect can occur congenitally or secondary to trauma, infective endocarditis, and other effects [1]. In humans, most indirect-type Gerbode defects are congenital defects [1]. Depending on the anatomical location of the shunt, Gerbode defect is classified into one of three types. Type 1 is a supravalvular defect, which occurs in the atrioventricular septum and forms between the LV and RA. Type 2 is an infravalvular defect that occurs between the ventricles. Type 3 , an intermediate defect, combines both supravalvular and infravalvular defects. In humans, the type 1 defect is more likely to be acquired while types 2 and 3 are usually congenital $[1,3]$.

In two dogs, Gerbode defects have been reported secondary to infective endocarditis and, in another two dogs, secondary to trauma [4-7]. All occurrences were classified as type 1 and direct. Our case is the first report of Gerbode defect in a cat, particularly an indirect type 2 defect accompanying a TV perforation. The etiology in this cat could not be clearly identified. The cat had a history of trauma, but was
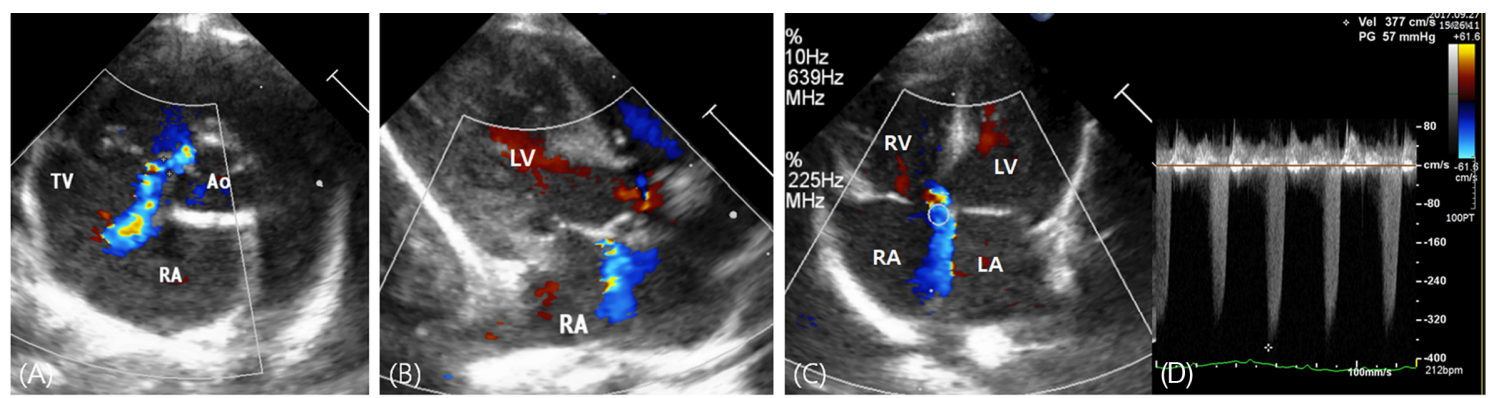

Fig. 3. Color-Doppler (A and B) and spectral-Doppler (C) echocardiography images; right short axis view (A), left cranial long axis view (B), and left apical long axis view (C). There was systolic eccentric turbulent flow in the RA (A and B). The turbulent flow was directed from the LV to the RV through the upper membranous septum and then it immediately flowed into the RA through the tricuspid septal leaflet. Shunt flow velocity was $3.77 \mathrm{~m} / \mathrm{sec}$ and the pressure gradient was $57 \mathrm{mmHg}$ (D).

Ao, aorta; LA, left atrium; LV, left ventricle; RA, right atrium; RV, right ventricle. 
asymptomatic for one month after the accident, unlike the previous two dogs that showed clinical signs within a few days of the trauma [4,5]. Another possible cause of a Gerbode defect, endocarditis, was not suspected in this cat because there was no vegetative valve change detected on echocardiography and no fever, although blood culture was not performed.

In this case, a tricuspid defect was suspected based on the direction of the shunt flow but the tricuspid defect could not be assessed due to the limitation of the cardiac window in conventional echocardiography. Although transesophageal echocardiography is more sensitive in detecting a tricuspid defect than transthoracic echocardiography, there is limited availability of this modality. In an indirect Gerbode defect, the VSD is located at a perimembranous area near the atrioventricular valves [8]. Therefore, turbulence generated from the VSD can be indistinguishable from the turbulent flow from the TV defect because the distance between the two turbulence is too short. If only turbulent flow around the TV is obvious it can be confused with tricuspid regurgitation (TR). Moreover, the turbulent flows in both diseases develop in the systolic phase and induce RA and RV enlargements. Based on a typical finding for the origin of the turbulent flow from the perimembranous septum rather than between the TV leaflets, Gerbode defect presence can be suspected [1]. However, in some cases, it can be difficult to differentiate between Gerbode defect and TR based on the direction of flow [9]. In that case, a comprehensive assessment of right-side cardiac changes may be helpful. Gerbode defects typically have very high velocities due to the high-pressure gradient between LV and RA. Thus, if Gerbode defect is confused with TR, pulmonary hypertension may be misdiagnosed. Gerbode defect should be considered when the case exhibits a high-velocity turbulent flow in the RA but is not accompanied by any evidence of pulmonary hypertension such as RV wall hypertrophy, pulmonary regurgitation, and pulmonary arterial enlargement.

The presence and severity of symptoms and the need for Gerbode defect treatment depend on the magnitude of the shunt, concomitant anatomical abnormalities, and comorbidities [1]. Over $25 \%$ of the congenital defects and $21 \%$ of the acquired defects in human patients were asymptomatic due to the small shunt volume $[1,8]$. Such asymptomatic patients can be monitored rather than undergo surgery [10].

Herein, the echocardiographic features of a Gerbode defect were described in the first case of an indirect type involving perimembranous VSD and TV perforation in a cat. When a high-velocity turbulent flow in the RA without pulmonary hypertension is observed on echocardiography, Gerbode defect presence should be considered. Careful assessment of origin, direction, and timing of the shunt flow can help in differentiating Gerbode defect from other conditions including TR.

\section{Acknowledgments}

This research was supported by the Basic Science Research Program of the National Research Foundation of Korea, which is funded by the Ministry of Science, ICT, and Future Planning (2018R1A2B6006775).

\section{References}

1. Breatnach CR, Walsh KP. Ruptured sinus of Valsalva aneurysm and Gerbode defects: patient and procedural selection: the key to optimising outcomes. Curr Cardiol Rep 2018;20:90.

2. Cunningham SM, Lindsey KJ, Rush JE. Acquired Gerbode defect and third-degree atrioventricular block secondary to vehicular trauma in a dog. J Vet Emerg Crit Care (San Antonio) 2013;23:637-642.

3. Garg R, Garcia R, Cubeddu RJ. Gerbode defect misinterpreted as pulmonary hypertension. J Cardiol Cases 2012;7: e34-e36.

4. Hezzell MJ, Dennis S, Lewis DH, Fuentes VL. Gerbode defect associated with blunt trauma in a dog. J Vet Cardiol 2011;13:141-146.

5. Peddle GD, Boger L, Van Winkle TJ, Oyama MA. Gerbode type defect and third degree atrioventricular block in association with bacterial endocarditis in a dog. J Vet Cardiol 2008; 10:133-139.

6. Ramírez GA, Espinosa de los Monteros A, Rodríguez F, Weisbrode SE, Jaber JR, Herráez P. Left ventricular outflow tract-right atrial communication (Gerbode type defect) associated with bacterial endocarditis in a dog. Vet Pathol 2003;40:579582.

7. Saker E, Bahri GN, Montalbano MJ, Johal J, Graham RA, Tardieu GG, Loukas M, Tubbs RS. Gerbode defect: a comprehensive review of its history, anatomy, embryology, pathophysiology, diagnosis, and treatment. J Saudi Heart Assoc 2017;29:283-292.

8. Silbiger JJ, Kamran M, Handwerker S, Kumar N, Marcali M. The Gerbode defect: left ventricular to right atrial communication-anatomic, hemodynamic, and echocardiographic features. Echocardiography 2009;26:993-998.

9. Sinisalo JP, Sreeram N, Jokinen E, Qureshi SA. Acquired left ventricular-right atrium shunts. Eur J Cardiothorac Surg 2011; 39:500-506.

10. Toprak C, Kahveci G, Akpinar S, Tabakçi MM, Güler Y. Concomitant Gerbode-like defect and anterior mitral leaflet perforation after aortic valve replacement for endocarditis. Echocardiography 2013;30:E231-E235. 